\title{
Pancreatic Mucinous-Cystic Neoplasm with Intermediate Grade Dysplasia
}

National Cancer Institute

\section{Source}

National Cancer Institute. Pancreatic Mucinous-Cystic Neoplasm with Intermediate

Grade Dysplasia. NCI Thesaurus. Code C6883.

A non-invasive mucinous cystic neoplasm that arises from the pancreas and is

characterized by the presence of moderate dysplasia. The neoplastic columnar mucinproducing epithelial cells form papillary projections or crypt-like invaginations. There is cellular pseudostratification and mitotic activity present. 